\title{
PREVALENCIA DEL STREPTOCOCCUS B EN EL TRACTO GENITAL INFERIOR EN EMBARAZADAS ENTRE 35 Y 37 SEMANAS
}

\section{HOSPITAL DE SAN JOSÉ}

José Luis Rojas Arias MD*, Marcela Patricia Pérez Pérez MD**, Edna Patricia Otálora MD**

\section{Resumen}

Introducción: la infección es la causa más importante de morbimortalidad en las unidades de cuidado intensivo neonatal a nivel mundial, por lo regular causadas por Streptococcus del grupo B. Objetivos: describir la prevalencia de colonización del germen en el tracto genital inferior de gestantes entre las semanas 35.0 y 37.6 en el Hospital de San José. Materiales y métodos: estudio descriptivo de corte transversal. Se realizaron cultivos vaginal y anorrectal a todas las pacientes, procesados de manera independiente y ante la positividad se administró profilaxis. Resultados: entre marzo 2008 y marzo 2009 se evaluaron 112 gestantes. La prevalencia del microorganismo fue 15,2\% (n=17) y la edad promedio fue de 28 años (DE 6.2). La actividad sexual en el tercer trimestre $(51,8 \%)$, la primiparidad $(48,2 \%)$ y el antecedente de vaginosis en III trimestre $(44,6 \%)$ fueron los factores observados con mayor frecuencia, sin encontrar asociación estadística significativa con la presencia del germen. Conclusiones: la prevalencia del Streptococcus $B$ fue similar a la reportada en otras publicaciones de países desarrollados o en vía de desarrollo. Teniendo en cuenta el impacto que tiene esta colonización del tracto genital inferior con desenlaces maternos y neonatales, debería considerarse la realización de esta prueba como tamizaje en pacientes gestantes en el tercer trimestre.

Palabras clave: embarazo, Streptococcus B, prevalencia.

\section{Abstract}

Introduction: worldwide, infection, usually caused by Group B Streptococcus, is the leading cause of morbidity/mortality at the neonatal intensive care units. Objectives: to describe the prevalence of colonization by this microorganism in the lower genital tract of pregnant women between weeks 35.0 and 37.6 at Hospital de San José. Materials and Methods: this is a cross sectional descriptive study. Vagina and rectum specimens were cultured for all patients, independently processed and prophylactic treatment was given when culture positive. Results: 112 pregnant patients were assessed between March 2008 and March 2009. Prevalence of the microorganism was $15.2 \%(n=17)$ and mean age was 28 years (SD 6.2). The most frequent factors were: sexual activity in the third trimester (51.8\%), primigravity (48.2\%) and a history of third-trimester vaginal infection (44.6\%), finding no statistically significant association with the presence of the studied germ. Conclusions: the prevalence of Group B Streptococcus infection was similar to that reported in literature of other developed or developing countries. The impact this colonization of the lower genital tract has on maternal and neonatal outcomes is an indication to recommend this test on third-trimester pregnant patients as a screening strategy.

Key Words: pregnancy, Group B Streptococcus, prevalence.

Fecha recibido: agosto 6 de 2009 - Fecha aceptado: febrero 15 de 2010

* Ginecoobstetra, especialista en Medicina Materno Fetal. Jefe del Servicio de Obstetricia Hospital de San José. Instructor Asistente, Funda- ción Universitaria de Ciencias de la Salud. Bogotá DC. Colombia.

** Residente IV año de Ginecología y Obstetricia de la Fundación Universitaria de Ciencias de la Salud. Bogotá DC. Colombia. 


\section{Introducción}

A lo largo de la historia la infección neonatal ha sido la principal causa de morbimortalidad en las unidades de recién nacidos a nivel mundial, incrementando las estancias hospitalarias por las implicaciones que conlleva. ${ }^{1}$ Cualquier medida encaminada a mejorar las estrategias de detección y manejo de los agentes causantes de dicha infección se verá reflejada en la disminución del ingreso de estos pacientes a la unidad de recién nacidos. En lo que se refiere a la sepsis de inicio temprano, se ha observado que el Streptococcus $B$ es una de las causas más importantes de infección neonatal severa (54\%) y muerte materna. ${ }^{1-4}$

Desde 1938 se conoce la existencia del Streptococcus $B$ o agalactiae como agente colonizador de la vagina y el recto en las mujeres embarazadas y causante de sepsis en el recién nacido. ${ }^{1,5}$ En la década de los ochenta varios estudios clínicos demostraron que la administración profiláctica de antibióticos durante el trabajo de parto en mujeres con riesgo de transmitir el germen a sus recién nacidos podría prevenir la enfermedad invasiva en la primera semana de vida neonatal. Por este motivo en 1996 el Colegio Americano de Obstetras y Ginecólogos (ACOG) el Centro de Prevención y Control de Enfermedades ${ }^{1}$ y en 1997 la Academia Americana de Pediatría $a^{2}$ promovieron una serie de recomendaciones para profilaxis intraparto encaminadas a la prevención de las infecciones perinatales atribuibles al microorganismo. ${ }^{3,6}$

En Colombia se sabe del impacto que representa la sepsis y neumonía intrauterina en el recién nacido pero se desconoce la prevalencia que tiene el germen. Se han realizado muy pocos estudios acerca del mismo y no es posible extrapolar datos a la población general. Además, no se han incluido estudios de tamizaje para detección de pacientes portadoras que puedan transmitir la infección a sus hijos, en las cuales se realizan estrategias de intervención temprana que disminuyen el riesgo de infección del $20 \%$ a menos del $1 \%$ según las tasas reportadas a nivel mundial y que muestran como las pruebas de tamizaje con intervención son $50 \%$ más efectivas en disminuir la infección neonatal que aquellas que sólo incluyen factores de riesgo para iniciar el tratamiento antibiótico. ${ }^{3}$ Basados en lo anterior se pretende realizar un estudio con el fin de conocer la prevalencia de colonización del
Streptococcus $B$ a nivel del tracto genital inferior en las mujeres con gestaciones entre las semanas 35 y 37 en el Hospital de San José de Bogotá.

\section{Métodos}

Estudio descriptivo de corte transversal que se llevó a cabo en el Hospital de San José durante el período comprendido entre marzo 1 de 2008 y marzo 1 de 2009. Se reclutaron gestantes que asistieron a la consulta externa de control prenatal con edad gestacional entre $35.0 \mathrm{y}$ 37.6 semanas. El protocolo fue aprobado por el Comité de Investigaciones y Ética de la Facultad de Medicina de la Fundación Universitaria de Ciencias de la Salud. Las gestantes que cumplían los criterios de inclusión fueron invitadas a participar en este estudio, se entrevistaron todas y se diligenció el instrumento de recolección de datos que tomó como fuente primaria aquella aportada de manera directa por la paciente y como secundaria la revisión de la historia clínica en busca de ingresos hospitalarios previos, tratamientos recibidos y otros antecedentes patológicos que no fueran bien conocidos por ella.

Se excluyeron aquellas con antecedentes de consumo de antibióticos por cualquier vía en los últimos quince días, presencia de sangrado vaginal en el momento de la toma del cultivo, uso de cremas, óvulos o duchas vaginales en los diez días previos al cultivo y ruptura de membranas en el momento de la toma de la muestra y/o en trabajo de parto. Se practicaron cultivos vaginal y anorrectal por la misma auxiliar de enfermería. La toma del cultivo se obtuvo con la gestante en posición ginecológica con apertura suave de labios mayores y menores para visualizar orificio vaginal sin utilizar espéculo. Las muestras fueron recolectadas con hisopo realizando frotis del tercio inferior de vagina (introito) y colocadas en tubo seco. Después se tomaron muestras a nivel anorrectal y colocadas también en tubos secos que se enviaron rotulados a la sección de microbiología del laboratorio. Se utilizaron medios de cultivo para gérmenes comunes que tienen sensibilidad del 90-95\%, que garantizan la viabilidad y aumentan la recuperación del germen. El reporte describe la sensibilidad a la penicilina y derivados.

En las pacientes con cultivo positivo para el microorganismo se realizó profilaxis antibiótica con ampicilina $2 \mathrm{~g}$ 
vía intravenosa iniciales y luego $1 \mathrm{~g}$ endovenoso cada cuatro horas hasta el momento del parto, previa prueba de sensibilidad al medicamento. En caso de alergia se administraría clindamicina $900 \mathrm{mg}$ IV cada ocho horas hasta el parto, de acuerdo a las recomendaciones del Centro de Control de Enfermedades en 2002. ${ }^{1,2}$ El análisis estadístico se realizó con el programa Stata $10^{\mathbb{R}}$. Las variables cuantitativas se reportan con medias y desviación estándar. Las variables categóricas con frecuencias absolutas y porcentajes. Para explorar asociación entre variables se utilizó la prueba $c h i^{2}$.

\section{Resultados}

Las 112 gestantes estudiadas tenían un rango entre 16 y 43 años con una media de 28 (DE 6.2), la edad gestacional al momento de la toma de la muestra fue de 35.1 a 37.4 semanas con una media de 36.5 (DE 0.6). Las características de la población relacionadas con la identificación de factores de riesgo propuestos por la literatura se presentan en la Tabla 1. En 17 pacientes se obtuvo cultivo positivo para Streptococcus B, doce $(10,7 \%)$ presentaron en ambos y cinco $(4,4 \%)$ sólo tuvieron positividad en el cultivo vaginal.

La prevalencia del germen en nuestra población fue $15,2 \%$. De las 17 positivas, 16 recibieron profilaxis antibiótica intraparto con ampicilina endovenosa. La sensibilidad para cefalosporinas y penicilinas fue del $100 \%$; sólo hubo resistencia a aminoglucósidos en tres $(17,6 \%)$. Aunque no fue el objetivo de este estudio pero hizo parte del protocolo de seguimiento del servicio, se evaluó el desenlace materno y perinatal adverso. El primero se presentó en dos casos con cultivos positivos y que desarrollaron endometritis puerperal. Se documentó uno de neumonía in utero $(0,89 \%)$ dentro del grupo de pacientes con ambos cultivos positivos.

\section{Posibles factores de riesgo}

La actividad sexual en el tercer trimestre $(51,8 \%)$, la primiparidad $(48,2 \%$ ) y el antecedente de vaginosis en el tercer trimestre $(44,6 \%)$ fueron los factores observados con mayor frecuencia en pacientes con cultivo positivo. De las 112 pacientes estudiadas nueve eran nulíparas,

\begin{tabular}{|c|c|c|}
\hline \multirow[b]{2}{*}{ Edad (promedio, DE) } & \multicolumn{2}{|c|}{$n=112$} \\
\hline & 28.0 & $(6.2)$ \\
\hline Edad gestacional (promedio, DE) & 36.5 & $(0.6)$ \\
\hline \multicolumn{3}{|l|}{ Nivel socioeconómico n (\%) } \\
\hline estratos 1 y 2 & 80 & (71.4) \\
\hline estratos 3 y 4 & 32 & (28.6) \\
\hline \multicolumn{3}{|l|}{ Paridad n (\%) } \\
\hline primigestante & 54 & $(48.2)$ \\
\hline multípara & 49 & $(43.8)$ \\
\hline nulípara & 9 & (8) \\
\hline \multicolumn{3}{|l|}{ Uso DIU n (\%) } \\
\hline sí & 23 & (20.5) \\
\hline no & 89 & (79.5) \\
\hline \multicolumn{3}{|l|}{ Antecedente de parto pretérmino $\mathrm{n}(\%)$} \\
\hline sí & 13 & (II.6) \\
\hline no & 46 & (4I) \\
\hline no aplica & 53 & $(47.4)$ \\
\hline Actividad sexual tercer trimestre $\mathrm{n}(\%)$ & 57 & $(51.8)$ \\
\hline \multicolumn{3}{|l|}{ Uso de preservativo ** $\mathrm{n}(\%)$} \\
\hline sí & 5 & (4.4) \\
\hline no aplica & 51 & $(45.6)$ \\
\hline \multicolumn{3}{|l|}{ RPM n (\%) } \\
\hline | y || trimestres & _- & - \\
\hline III trimestre & - & - \\
\hline Antecedente de IVU III trimestre n (\%) & 30 & $(26.7)$ \\
\hline Antecedente de vaginosis $\mathrm{n}(\%)$ & 50 & $(44.6)$ \\
\hline Fiebre $\mathrm{n}(\%)$ & 7 & (6.2) \\
\hline
\end{tabular}

54 primigestantes y 49 multíparas; ocho de las primigestantes cursaron con ambos cultivos positivos (66,6\% del total de positivos). La Tabla 2 presenta las características de las gestantes según el resultado del cultivo. Al realizar la prueba $c h i^{2}$ no se encontró asociación estadística significativa para ninguno de los factores de riesgo descritos en la literatura y la presencia del germen en la población estudiada.

\section{Discusión}

En Colombia y en especial en Bogotá se desconoce la prevalencia real del Streptococcus B. En el Hospital de San José de Bogotá es el primer estudio que se rea- 


\begin{tabular}{|c|c|c|c|c|c|}
\hline & \multicolumn{2}{|c|}{$\begin{array}{c}\text { Cultivo }(+) \\
\quad(n, \%)\end{array}$} & \multicolumn{2}{|r|}{$\begin{array}{c}\text { Cultivo (-) } \\
(\mathrm{n}, \%)\end{array}$} & \multirow[t]{2}{*}{$\mathrm{P} * *$} \\
\hline & 17 & $(15,1 \%)$ & 95 & $(84,9 \%)$ & \\
\hline \multicolumn{6}{|l|}{ Edad materna } \\
\hline menores de 28 años & 10 & $(58,8)$ & 41 & $(43, I)$ & NS \\
\hline mayores o igual a 28 años & 7 & $(4 I, 2)$ & 54 & $(56,9)$ & \\
\hline \multicolumn{6}{|c|}{ Edad gestacional al momento de toma de la muestra } \\
\hline semanas mediana (RIQ) & 36.3 & $(36.1-37)$ & 37 & $(36.1-37)$ & \\
\hline \multicolumn{6}{|l|}{ Nivel socioeconómico } \\
\hline estratos 1 y 2 & 12 & $(70,6)$ & 68 & $(7 I, 5)$ & NS \\
\hline estratos 3 y 4 & 5 & $(29,4)$ & 27 & $(28,4)$ & \\
\hline \multicolumn{6}{|l|}{ Paridad } \\
\hline primigestante & 10 & $(58,8)$ & 44 & $(46,3)$ & NS \\
\hline multípara & 5 & $(29,4)$ & 44 & $(46,3)$ & \\
\hline nulípara & 2 & $(11,8)$ & 7 & $(7,4)$ & \\
\hline Uso de DIU * & 2 & $(I I, 8)$ & 21 & $(22, I)$ & NS \\
\hline Antecedente de parto pretérmino † & 1 & $(5,9)$ & 12 & $(12,6)$ & NS \\
\hline Actividad sexual en III trimestre & 6 & $(35,2)$ & 51 & $(54,8)$ & NS \\
\hline Uso de preservativo $\dagger \dagger$ & 0 & - & 5 & $(5,3)$ & NS \\
\hline Fiebre & $\mathrm{I}$ & $(5,9)$ & 6 & $(6,3)$ & NS \\
\hline Antecedente de IVU en III trimestre & 3 & $(17,7)$ & 27 & $(28,4)$ & NS \\
\hline Antecedente de vaginosis & 7 & $(4 I, 2)$ & 43 & $(45,3)$ & NS \\
\hline
\end{tabular}

* DIU: dispositivo intrauterino, † parto pretérmino antecedente, $\nmid \dagger$ uso de preservativo como método de protección en tercer trimestre de gestación actual, ** todos los valores $\mathrm{p}$ obtenidos a partir de prueba de chi ó test exacto de Fischer para asociación, NS no significativo $\mathrm{p}>0,05$.

liza acerca de este tópico y que contribuirá a la disminución de la infección materna y neonatal en la institución, gracias al conocimiento que se adquirió acerca de la presencia de infección en las pacientes con cultivos positivos. La población objeto de estudio fue el total de la que acudió al servicio de ginecología durante un período de un año y que cumplió con los criterios de inclusión establecidos, lo cual constituye una fortaleza importante pues no requirió muestreo, como también lo fue el rigor con que se vigilaron todos los datos y se llevó a cabo el proyecto.

Es el microorganismo más importante cuando se analizan los casos de sepsis neonatal precoz de origen bacteriano, infección neonatal y materna, desplazando al segundo lugar a $E$. coli. ${ }^{7,8}$ Por esta razón, desde hace más de doce años diversos centros en el mundo han su- gerido estrategias para prevenir esta patología. Se ha demostrado que la mejor intervención para reducir la incidencia de estos eventos es la aplicación de antibióticos durante el trabajo de parto. En esto coinciden todos los protocolos vigentes ${ }^{7,9}$ y en lo que difieren es en la selección del grupo de embarazadas a quienes se les aplicará dicha intervención. En 1992, la Academia Americana de Pediatría $(A A P)$ recomendó tamizaje universal a las 26 a 28 semanas de gestación y tratar intraparto a las pacientes con factores de riesgo y que tuviesen cultivo positivo. Ese mismo año, el Colegio Americano de Obstetricia y Ginecología ( $A C O G$ ) recomendó tratar a las pacientes sólo basados en factores de riesgo, sin realizar tamizaje. ${ }^{5,7,10}$ En 1996 el Comité del ACOG comentó que las recomendaciones pediátricas y obstétricas de 1992 producían controversias y confusiones entre los clínicos. Por ello, el CDC de EE.UU., también en 1996, 
planificó dos estrategias posibles y sugirió que cada centro de salud analizara de acuerdo a su realidad local cual es la más apropiada. ${ }^{7}$ Una se basa en administrar antibióticos intraparto sólo a las pacientes con los factores de riesgo y la otra agrega el tamizaje universal entre las 35 y 37 semanas de gestación para la población de bajo riesgo. ${ }^{5,7,10}$ En la actualidad está vigente la recomendación del $C D C$ del 2002, revisada en 2006, con tamizaje universal y cultivos a todas las pacientes embarazadas. ${ }^{411}$ Las incidencias internacionales de desenlaces neonatales y maternos adversos más bajas reportadas a la fecha, en su mayoría corresponden a centros que están aplicando esta última recomendación. Sin embargo, es importante destacar que a pesar de estas pautas conocidas a nivel mundial, en el país no se han establecido lineamientos por parte del Ministerio de Protección Social y las Secretarías de Salud para el tamizaje del germen en la población embarazada, aún conociendo el impacto de su colonización.

Los estudios de prevalencia del microorganismo en embarazadas muestran tasas de colonización que varían entre el 5 y $25 \%{ }^{6,12-17}$ dependiendo de la población estudiada, la ubicación geográfica, la región anatómica de obtención de la muestra (vaginal y/o anal) y el medio de cultivo utilizado (selectivo o no). La prevalencia del germen en este estudio fue $15,2 \%$, similar a la reportada en otras publicaciones de países desarrollados o en vía de serlo. ${ }^{1,2,14,18-20}$ Este estudio permite enfatizar la necesidad del uso de medios de cultivos selectivos en la pesquisa del germen, dado que en las poblaciones gestantes de similares características demográficas, la prevalencia utilizando un medio no selectivo fue de $6,2 \% \mathrm{y}$ al introducir uno selectivo subió a $14 \%$. $^{6,8,11,21,22} \mathrm{El}$ caldo de cultivo Todd Hewitt como medio selectivo cumple con las exigencias necesarias para el desarrollo del microorganismo ya que cuenta en su composición con ácido nalidíxico y gentamicina, antibióticos que inhiben el desarrollo de otros gérmenes y que pueden interferir en el aislamiento. ${ }^{9,23-26}$ De igual manera, este estudio ratifica la importancia de la toma de cultivos de la región vaginal y perianal, ya que en un porcentaje importante se aisló sólo en la muestra vaginal (29,4\%), lo que significa que la toma perianal única deja cerca de un tercio de las embarazadas en riesgo de transmisión vertical al no recibir profilaxis.
El esfuerzo para detectar el germen tiene como fin disminuir la incidencia de sepsis neonatal precoz y de infección neonatal y materna, para lo cual cada institución debe contar con una adecuada profilaxis antibiótica para sus embarazadas durante el trabajo de parto. Esto hace necesario conocer la sensibilidad antibiótica del germen en esta población. En este estudio todas las cepas aisladas presentaron sensibilidad a ampicilina, antibiótico sugerido como tratamiento de elección. ${ }^{27-30}$

La infección neonatal y materna tiene un gran impacto en salud pública debido a la elevada tasa de mortalidad sobre todo neonatal ( 58 a 71\%), lo único que ha demostrado efectividad es la administración de antibióticos desde el inicio del trabajo de parto. Como ya se mencionó, la estrategia más efectiva es la que considera a las embarazadas con factores de riesgo y la población de bajo riesgo con cultivos positivos y que son tratadas de manera profiláctica durante el trabajo de parto. ${ }^{5,7,10}$ En nuestra población, la actividad sexual en el tercer trimestre, la primiparidad y el antecedente de vaginosis en ese mismo trimestre fueron los factores observados con mayor frecuencia en pacientes con cultivo positivo, pero no se demostró una asociación estadística significativa entre la presencia de estos factores de riesgo y el resultado de los cultivos.

Aunque una de las limitaciones de este estudio fue el diseño que no incluía evaluación de desenlaces maternos y neonatales, éstos fueron explorados aún sin ser uno de los objetivos. Los resultados obtenidos nos permiten observar que las pacientes con cultivo positivo y presencia de factores de riesgo podrían tener algún tipo de relación con el desenlace adverso tanto materno como neonatal. En la población con cultivos positivos se observaron con mayor frecuencia desenlaces maternos adversos como endometritis, sin poder afirmar que este hallazgo sea predictivo puesto que este tipo de estudio no lo permite. La alta prevalencia de portadores del microorganismo en la población estudiada justifica continuar con el método de tamizaje prenatal. Además, se debe considerar el impacto que tiene la colonización del tracto genital inferior en gestantes y la posibilidad de reducción de morbimortalidad por infección neonatal o materna mediante el aislamiento bacteriológico entre las 35 y 37 semanas de gestación. ${ }^{31,32}$ 
Concluimos que el tamizaje debe realizarse a todas las mujeres gestantes en las semanas 35 a 37 , mediante cultivos selectivos tomados de la vagina y región perianal, con el posterior tratamiento antibiótico profiláctico de las portadoras al ingreso en trabajo de parto. Este estudio debe fortalecer líneas de investigación en el departamento de ginecología y obstetricia que puedan seguir aportando conocimientos y que conduzcan al establecimiento de conductas adecuadas para el diagnóstico y tratamiento de este germen.

\section{Referencias}

1. Prevention of early-onset group B streptococcal disease in newborns. Int $\mathrm{J}$ Gynaecol Obstet. 2003 Apr;81(1):115-22

2. Prevention of Perinatal group B streptococcal disease: a public health perspective. MMWR Recomm Rep. 1996 May 31;45(RR-7):1-24

3. Revised guidelines for prevention of early-onset group B streptococcal (GBS) infection. American Academy of Pediatrics Committee on Infectious Diseases and Committee on Fetus and Newborn.. Pediatrics 1997; 99(3):489-96.

4. Trends in Perinatal Group B Streptococcal Disease. United States, 20002006. MMWR Morb Mortal Wkly Rep. 2009 Feb 13;58(5):109-12

5. Decreasing incidence of perinatal group B streptococcal desease - United States, 1993-1995. MMWR Morb Mortal Wkly Rep. 1997 May $30 ; 46(21): 473-7$

6. Valdés E, Pastene C, Grau M, Catalán J, Candia P, Juarez G, Caballero R Prevalencia de colonización por Streptococcus Agalactiae (grupo B) en el tercer trimestre del embarazo en medio de cultivo no selectivo. Rev Chil Obstet Ginecol. 2003, 68(4): 305-08

7. Adoption of hospital pólices for prevention of perinatal group B streptococcal disease. United States, 1997. MMWR Morb Mortal Wkly Rep. 1998 Aug $21 ; 47(32): 665-70$.

8. Crespo MP, Vélez JD. Importancia clínica del Streptococcus Agalactiae como causante de infección. Colomb Med. 1996; 27(2): 53-58.

9. Argentina. Ministerio de Salud y Ambiente. Dirección Nacional de Salud Materno Infantil. Recomendaciones para la prevención, diagnóstico y tratamiento de la infección precoz por estreptococo B hemolítico del grupo $\mathrm{B}$ (EGB). Buenos Aires : El Ministerio; 2004

10. Main EK, Slagle T. Prevention of early-onset invasive neonatal Group streptococcal disease in private hospital setting: the superiority of culturebased protocols. Am J Obstet Gynecol 2000; 182(6): 1344-54

11. Regan JA, Klebanoff MA, Nugent RP et al. Colonization with group B streptococci on pregnancy an adverse outcomes. Am J Obstetric Gynecol $1996 ; 174: 1354-1360$.

12. Cortés H. Prevención de la infección neonatal por estreptococo del grupo B, ¿Es necesaria en nuestro medio? Rev. Col Obs. Gin. 2005; 56:231-238.

13. Gibbs R, Sweet R. Materna land fetal infectious disorders. En: Creasy R, Resnik R. Maternal-fetal medicine. 4a. ed. Philadelphia: Saunders; 2004. p 674-77.
14. Restrepo A, Serna L, Vanegas C, et al. Prevalencia de Streptococcus agalactiae en gestantes con factores de riesgo y sus recién nacidos. Hospital Universitario San Vicente de Paul, 2002. Infectio $2003 ; 7(3)$ : 147-52.

15. Trujillo M, Ospina B, Fama M. Reevaluación del estado de colonización por streptococcus del grupo B en madres e hijos al momento del parto. Rev Ces Medicina. $1999 ; 13: 44$.

16. Manotas RJ, Baquero D. Frecuencia de aislamiento de Streptococcus agalactiae en un grupo de embarazadas y sus productos. IATREIA $1989 ; 2$ :111-13

17. González CP, González JE. Prevalencia de Estreptococo Beta hemolítico en mujeres embarazadas de alto riesgo en el Hospital Simón Bolivar de Bogotá UCIN. 2001; 2 :7-15

18. Picard F J, Bergeron M G. Laboratory detection of group B Streptococcus for Prevention of perinatal disease. Eur J Clin Microbiol Infect Dis. 2004; 2 : 665-71.

19. Schray SJ, ZywicK S, Farley MM, et al. Group B Streptococcal disease in The era of intrapartum antibiotic prophylaxis. N Engl. J Med. 2000; 342: 1520 .

20. Duran KS, Chang, Benoit VM, et al. Group B streptococcal B-hemolysin/ cytolisin promotes invasion of human lung epithelial cells and the release of interleukin-8. J Infect Dis. 2002 Jan 15;185(2):196-203

21. Mandell GL, Benneth JE, et al. Streptococcus agalactiae (group B Streptococcus). In: Principles and practice of Infectious Diseases. 6th ed. Philadelphia : Churchill Livingstone; 2005. 199:2423-2434

22. Regan JA, Klebanoff MA, Nugent RP. The epidemiology of group B streptococcal colonization in pregnancy. Vaginal infections and prematurity Study Group. Obstet Gynecol 1991; 77(4):604-10

23. Meyn LA, Moore DM, Hillier SL, et al. Association of sexual activity with colonization and vaginal acquisition of group B Streptococcus in non pregnant women. Am J Epidemiol. 2002; 155: 949-957.

24. Campbell JR, Hillier SL, et al. Group B Streptococcal colonization and serotype-specific immunity in pregnant women at delivery. Obstet Gynecol. 2000; 96: 498-503

25. Baker CJ, Edwards MS. Group B streptococcal infections. In: Remington JS, Klein JO, eds. Infectious diseases of fetal and newborn infants. 5 th ed. Philadelphia: Saunders, 2000: 1091-156

26. Farley MM. Group B streptococcal disease in non pregnant adults. Clin infect Dis. 2001; 33: 556-61.

27. Bergeron MG, Menard C, et al. Rapid detection of Group B streptococci in pregnant women at delivery. N Engl. J Med. 2000; 343: 175-79

28. Kong F, et al. Serotype identification of Group Streptococci by PCR and sequencing. J Clin Microbiol. 2002; 40: 216-26

29. American Academy of Pediatrics. Group B streptococcal infections. In Pickering LK, ed. Red Book: report of the committee on Infectious Diseases. 26th ed. Washington: American Academy of Pediatrics; 2003. p. 584-91.

30. Bergeron M, Danbing K, Menard C, Picard F, Gagnon M, Bernier M, Ouellette M, Roy PH, Marcoux S, Fraser WD (2000) Rapid detection of group B streptococci in pregnant women at delivery. N Engl. J Med. 2009;343(3):175-79.

31. Colombia. Ministerio de Salud. Resolución No. 008430. Octubre 4 de 1993. Normas científicas, técnicas y administrativas para la investigación en Salud.

32. Cortés H. Prevención de la infección neonatal por estreptococo del grupo B ¿Es necesaria en nuestro medio?. Rev. Col Obs. Gin. 2005; 56:231-38. 